PREPRINT

AS54

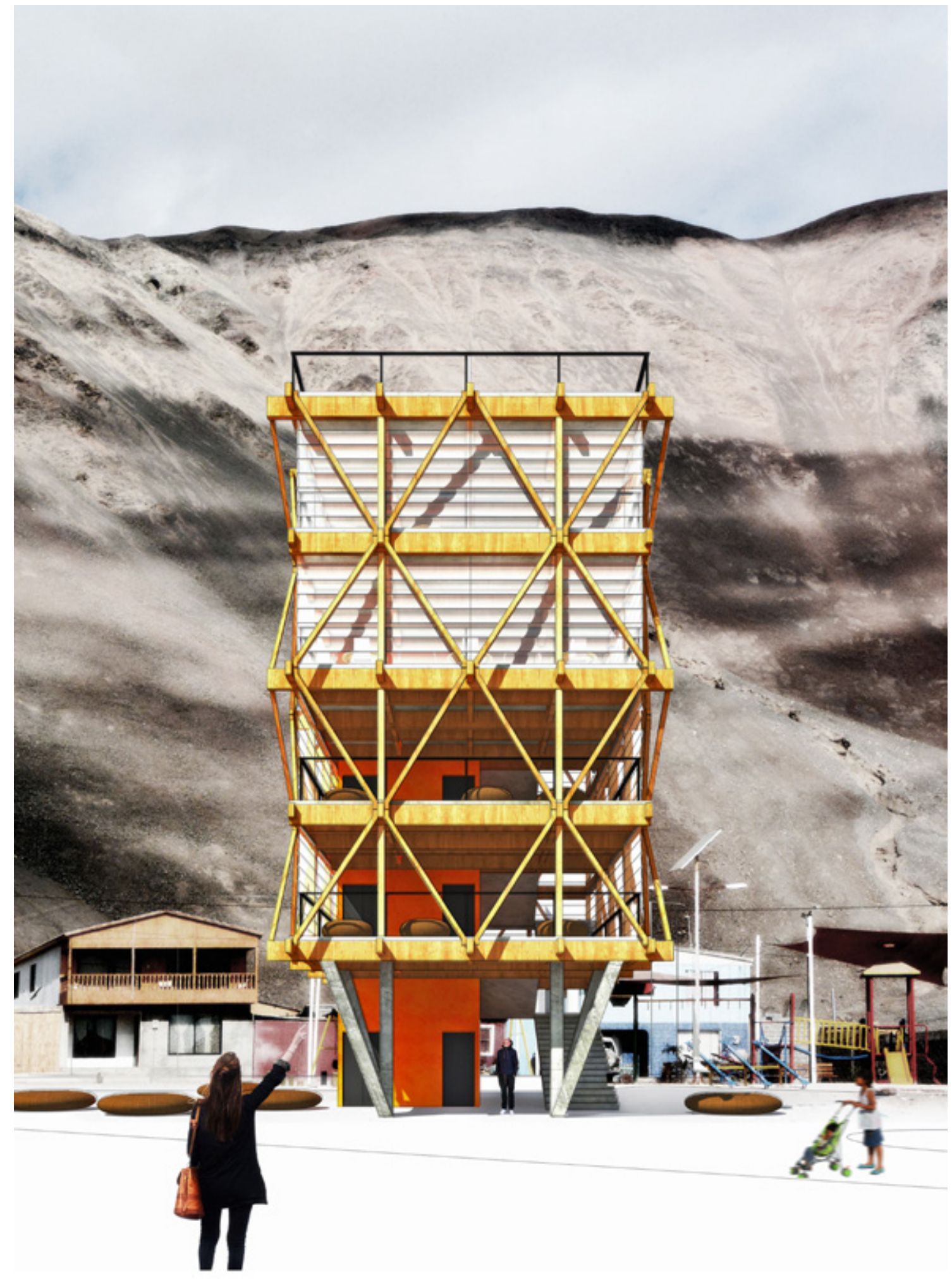



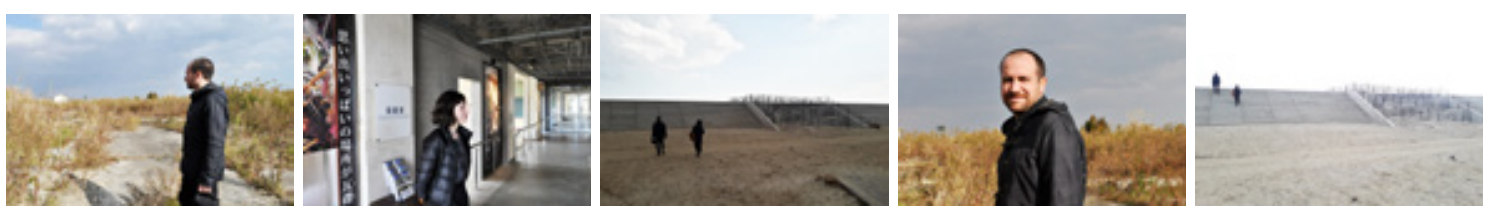

\title{
ARQUITECTURA Y EMERGENCIA: SISTEMA DE EVACUACIÓN VERTICAL PARA IQUIQUE, CHILE'
}

\author{
ARCHITECTURE AND EMERGENCY: A VERTICAL EVACUATION SYSTEM FOR IQUIQUE, \\ CHILE $^{1}$
}

\author{
Sebastian Laclabère Arenas $^{2}$, Claudia Oliva Saavedra ${ }^{3}$
}

RESUMEN

\begin{abstract}
Chile posee una larga historia de terremotos y tsunamis, y como resultado, el país ha implementado un estricto sistema normativo que ha permitido dar una respuesta adecuada a casos de terremotos recientes. Ahora bien, en caso de tsunami, tales respuestas se encuentran aún en una etapa de desarrollo, particularmente después de los sucesos del año 2010. La ciudad de lquique presenta un serio desafío en ese sentido, por cuanto extensas áreas podrían sufrir serios daños, a causa de inundaciones de 5 a 10 metros. Por otra parte, en muchos sectores la distancia entre la costa y las zonas de seguridad es excesiva, lo que presenta serios riesgos para la población. Este artículo examina lquique y sus potenciales escenarios de tsunami, identificando y localizando las debilidades de la red urbana de seguridad. Posteriormente, se exploran posibles mejoras, desde el punto de vista del diseño arquitectónico, concretamente a través de la incorporación de un sistema de estructuras de evacuación vertical.
\end{abstract}

Palabras clave: desastres naturales, tsunami, infraestructura, resiliencia, diseño urbano.

\section{ABSTRACT}

Chile has a long history of earthquakes and tsunamis, and as a result the country has implemented a strict regulatory system that has enabled adequate responses in the case of recent earthquakes. However, responses to tsunami events are still in a developmental stage, especially after the earthquake and tsunami of 2010. In this sense, the city of Iquique presents a serious challenge with regard to tsunami, as it could suffer severe, extensive damage caused by flooding of 5-10 meters. Moreover, in many places the distance between the shore and safe areas on higher ground is excessive, thereby causing serious risk for the population. This article examines lquique and its potential tsunami scenarios by identifying and pinpointing the weaknesses in its urban safety network. Finally, possible improvements to the existing system are explored from an architectural design viewpoint, specifically through the incorporation of a system of vertical evacuation structures.

Keywords: natural disasters, tsunami, infrastructure, resilience, urban design.

Artículo recibido el 14 de septiembre de 2018 y aceptado el 3 de diciembre de 2018 DOI: https://doi.org/10.22320/07196466.2018.36.054

[1] Este articulo se basa en los resultados de la investigación de la Tesis de Master in Engineering mention in Urban Design del autor titulada "Architecture + Emergency: Urban Safety Network in Iquique, Chile", Department of Architecture and Building Science, Tohoku University, Sendai, Japón.

[2] Research Student, Universidad de Tohoku, Sendai, Japón. sebastian.laclabere@gmail.com

[3] Research Student, Universidad de Tohoku, Sendai, Japón. claudiaoliva.arq@gmail.com 


\section{INTRODUCCIÓN}

Debido a su localización a lo largo del "Anillo de fuego del Pacífico" y sus caracteristicas geográficas, Chile ha estado constantemente afectado por sucesos sísmicos a lo largo de su historia (Barrientos, 2007). Esto ha influenciado de gran manera el desarrollo cultural y técnico de nuestro país, pasando a ser parte de nuestra identidad y arraigo. Como respuesta a esta situación, en las últimas décadas el desarrollo de la ingeniería, el diseño urbano y la arquitectura han alcanzado un nivel de preparación que ha permitido al país resistir de buena manera sismos de gran magnitud en los últimos años, incluyendo el terremoto del $27 \mathrm{~F}$ en 2010 (magnitud $8.8 \mathrm{Mw}$ ) y el de lquique, en el año 2014 (magnitud $8.2 \mathrm{Mw})$.

Por otra parte, estos mismos sucesos recientes han puesto en jaque la preparación de Chile frente a eventos de tsunamis, con grandes daños y pérdidas humanas tras el citado terremoto de $27 \mathrm{~F}$, en particular en localidades como Constitución, Talcahuano y Juan Fernández, entre otros. A partir de entonces, se han realizado grandes esfuerzos por parte del Estado y sus instituciones por perfeccionar el rendimiento de las ciudades costeras de Chile frente a los tsunamis, especialmente a través del diseño de normativas técnicas específicas para estos fenómenos y la elaboración de planes de reconstrucción interdisciplinarios denominados PRES, enfocados en generar asentamientos más sustentables y resilientes para enfrentar futuros sucesos de similares caracteristicas. 


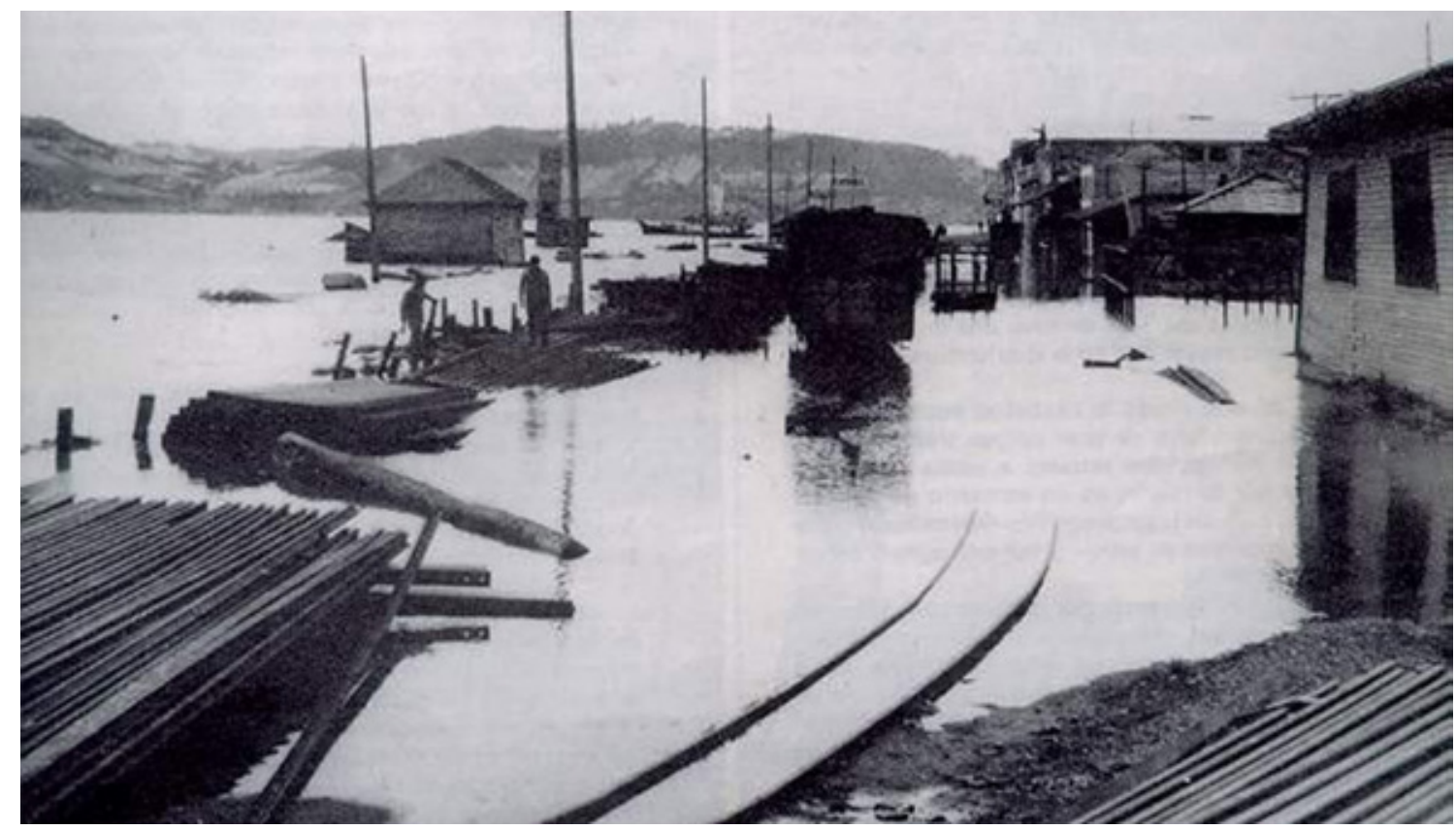

Figura 1 Vista del puerto de Castro tras el terremoto de Valdivia 1960 Fuente: Archivo Histórico y Bibliográfico de Chiloé (autor desconocido).

Teniendo estas consideraciones de fondo, los objetivos de este artículo serán entender la realidad de Chile con respecto a sismos y tsunamis, observar a modo de ejemplo la situación de lquique como posible escenario de futuros eventos y explorar potenciales contribuciones a la preparación de las ciudades costeras en Chile, desde el punto de vista del diseño arquitectónico.

\section{Terremotos en Chile: Historia y regulaciones}

Como ya ha sido mencionado, Chile posee una larga y constante historia de sucesos sísmicos y tsunamis, cuyos primeros datos se registran a partir de la época de la colonia, en el año 1647. Algunos de los eventos sísmicos más notables y destructivos en la historia del planeta han tenido lugar en Chile, como el terremoto de Vallenar de 1922, de una magnitud de $8.5 \mathrm{Mw}$, el terremoto de Valdivia, el más grande jamás registrado, de una magnitud de 9.5Mw (Figura 1), y el reciente y altamente destructivo terremoto del $27 \mathrm{~F}$, de una magnitud de $8.8 \mathrm{Mw}$, que azotó toda el área centro-sur del país y provocó un alto impacto en términos económicos y humano ${ }^{4}$. Esta sucesión constante de desastres naturales ha ayudado a construir y dar forma a una expresión urbana y arquitectónica única, como respuesta a las demandas particulares de nuestro territorio y sus embates.

Considerando este contexto de constantes desastres naturales, los marcos regulatorios y técnicos en Chile se han modificado de manera progresiva a través de los años, apuntando cada vez a un estándar más exigente y a una mejor respuesta frente a estos sucesos. La primera aproximación a una normativa arquitectónica y urbana aparece con la "Ley General de Urbanismo" del año 1929, seguida prontamente por el “DFL 345 Ley de Construcciones y Urbanización" del año 1935; pero éstas aún enfrentan el problema desde una perspectiva bastante general, en tanto la primera regulación específicamente sismo resistente aparece en el año 1972, como finalización de un esfuerzo de mejoras regulatorias iniciadas doce años antes, en el año 1960, como respuesta al terremoto de Valdivia, a través de la NCh433of72. 5

Las regulaciones sísmicas en Chile, por lo general, van de la mano del más reciente desastre y buscan optimizarse sobre los problemas detectados en esos momen- 
tos, de modo que las últimas modificaciones importantes fueron realizadas en 2010, después del terremoto del 27 de febrero (Herrman, 2016). En esta ocasión no solo se consideraron mejoras a las regulaciones de terremotos, sino también, y por primera vez, se incluyó una serie de normativas específicas para situaciones de tsunami, nunca antes consideradas de manera tan protagónica dentro de las normativas nacionales.

Después de los terremotos de 2010 y 2014, se ha notado un esfuerzo por acentuar la discusión en torno a la vulnerabilidad de nuestras ciudades y la necesidad de generar nuevas herramientas que nos permitan estar mejor preparados hacia el futuro (Baeriswyl, 2014). Algunos ejemplos de iniciativas derivadas de dichas discusiones son el desarrollo de una serie de 27 Planes de reconstrucción sustentable (PRES), que buscan reconstruir y mejorar las condiciones generales de las ciudades más afectadas por el terremoto y tsunami del 27F. Estos planes buscan no solo incrementar la resiliencia en caso de desastre, sino también las condiciones de vida y la sustentabilidad en estos asentamientos. Asimismo, se han desarrollado propuestas con un carácter preventivo, enfocadas no solo a las ciudades afectadas durante el $27 \mathrm{~F}$, sino además a ciudades que no han sido aún aquejadas por eventos de este tipo. De esa forma, se ha puesto el foco en la mejora de la calidad y el acceso a la información por parte de la comunidad, la elaboración de planos de seguridad y evacuación para todas las ciudades costeras del país, y la realización de simulacros de evacuación en varias de las más importantes zonas costeras.

Resulta importante recalcar que a pesar de que la discusión y el trabajo con respecto a los desastres naturales en las áreas urbanas han aumentado, la efectividad de las herramientas de planificación territorial y el actual modelo de gestión de las ciudades siguen bajo cuestionamiento. Se hace imperante, entonces, considerar la variable "riesgo" en todas las futuras herramientas de planificación y gestión urbana (Baeriswyl, 2014).

\section{Contexto sísmico del norte de Chile}

En años recientes, varios estudios han sugerido la posibilidad latente de un terremoto de gran escala en la zona norte del país, específicamente en las regiones de Tarapacá y Arica y Parinacota. La mayor razón que explica la posibilidad de este suceso es la existencia de una laguna sismica entre el norte de Chile y el sur de Perú, que presentó un quiebre notable por última vez el año 1877 , producto de un gran terremoto de magnitud $8.8 \mathrm{Mw}$ que rompió desde el sur de Arica hasta la península de Mejillones.

El intervalo de recurrencia para terremotos de gran escala en este sector para los últimos 500 años está estimado en periodos de 111-144 años, convirtiendo a este sector en el área con la laguna sísmica de mayor antigüedad a lo largo de toda la Placa Sudamericana. A esto se suma que en las últimas dos décadas, los dos segmentos adyacentes al norte y sur de este sector han presentado terremotos de gran magnitud: el de $8.1 \mathrm{Mw}$ en Antofagasta el año 1995, y el 8.4Mw, en Arequipa, sector sur de Perú, el año 2001 (Schurr et al., 2014).

En 2007, a través de una iniciativa internacional, se estableció un centro de monitoreo, creado por una serie de universidades e instituciones, tanto chilenas como europeas, denominado IPOC (Integrated Plate Boundary Observatory). Este esfuerzo de monitoreo conjunto tiene como objetivo el estudio y la observación de esta zona en pos de enriquecer el conocimiento de esta área y la preparación para enfrentar posibles sucesos sísmicos de gran magnitud.

Debido a su gran extensión, esta región vulnerable concentra un número considerable de asentamientos, desde pueblos de menor escala a centros urbanos mayores, incluyendo dos de las más importantes ciudades del país, Iquique y Arica, que concentran una población que sobrepasa los 550.000 habitantes, según datos del último censo ${ }^{6}$.

\section{Caso de estudio: Iquique}

Como caso de estudio se selecciona la ciudad de lquique, capital de la Región de Tarapacá, en el extremo norte del país, que se vio afectada por un terremoto de magnitud $8.0 \mathrm{Mw}$ y un tsunami de escala menor en el año 2014, y que presenta un desafío particularmente complejo con respecto a su respuesta ante un potencial tsunami. Iquique se localiza a lo largo de una angosta planicie costera, de manera adyacente a la Cordillera de la Costa, que en este sector presenta farellones con alturas sobre los $600 \mathrm{msnm}$; su clima es desértico costero, seco y caluroso, con casi inexistente presencia de precipitaciones. La ciudad posee una población de 180,601 habitantes, según datos del último censo, en tanto la vecina comuna de Alto Hospicio registra 112,142 habitantes, con lo cual la población total del denominado "Gran lquique" (conurbación lquique-Alto Hospicio) pasa a bordear los 300,000 habitantes.

La ciudad también presenta un carácter singular en términos urbanos y arquitectónicos, siendo su expresión fuertemente influenciada por el rol de importante ciudad portuaria y minera, que cumplió durante el auge salitrero del siglo XIX y el gran número de inmigrantes recibidos a causa de dichas actividades económicas (Gurovich, 2005). Estas caracteristicas derivaron en el desarrollo de una arquitectura única basada en la adaptación de sistemas importados como el "balloon frame" norteamericano al clima desértico y a ciertas pre existencias, en términos de trazado urbano, como la fachada continua (Pizzi, 2005). En las últimas décadas, la ciudad se ha transformado en un importante núcleo turístico y comercial, no solo a nivel nacional sino también a nivel sudamericano. 


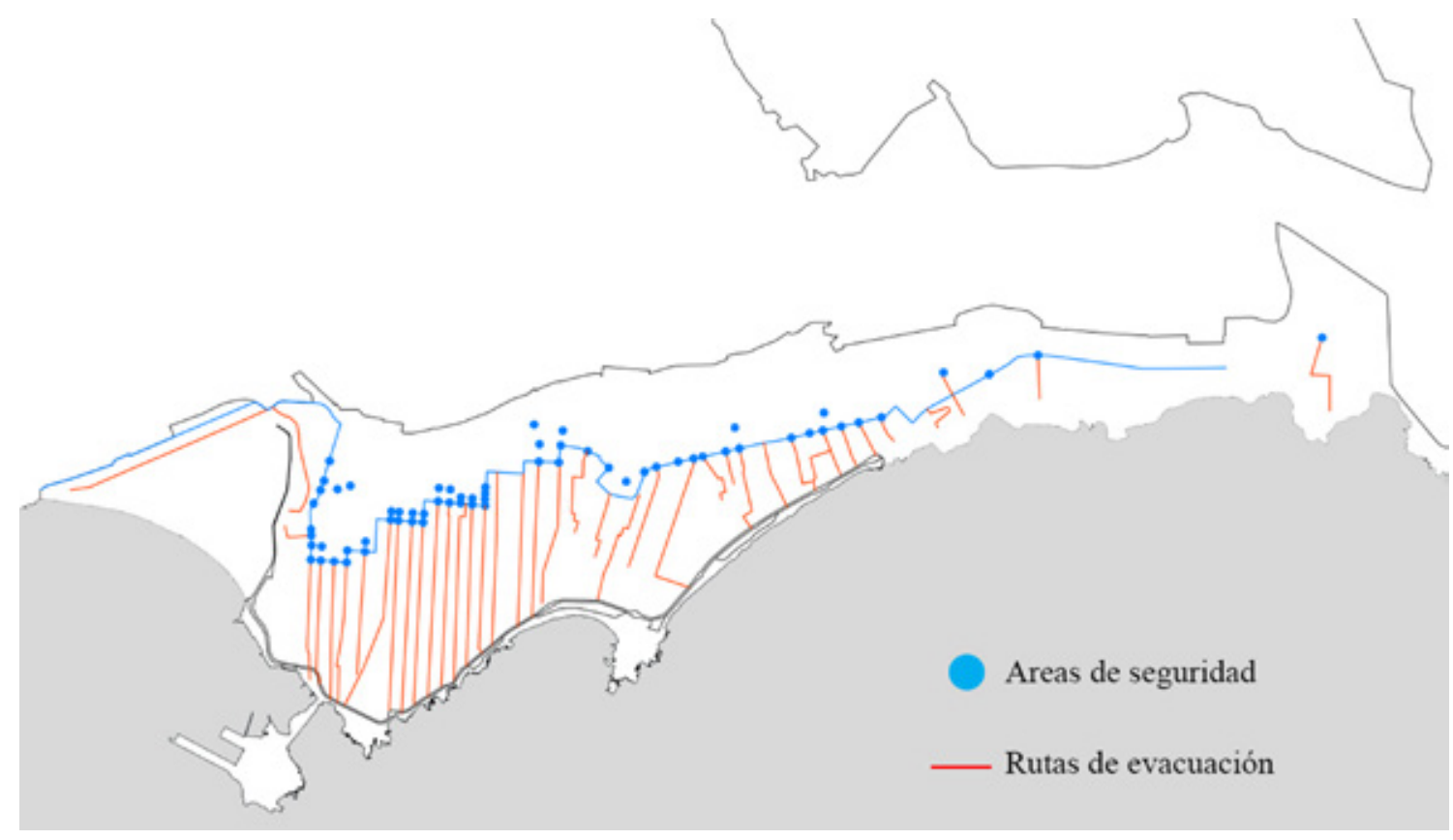

Figura 2 Situación actual red urbana de seguridad en Iquique, que considera rutas de evacuación, línea y zonas de seguridad definidos por ONEMI. Fuente: Elaboración de los autores.

\section{MÉTODO}

Este capítulo se centra en el análisis del caso de estudio, la ciudad de lquique, y en la evaluación de su red de seguridad urbana, principalmente a través del examen de mapas de potenciales inundaciones y tiempos de evacuación hipotéticos en caso de tsunami, desarrollados por investigaciones anteriores. Posteriormente, se propone la incorporación de una nueva variante, consistente en un sistema de estructuras de evacuación vertical, que podría optimizar la performance de la ciudad en caso de desastres naturales $y$, al mismo tiempo, mejorar las condiciones actuales de calidad de vida de sus habitantes.

\section{Red de seguridad urbana existente}

Como consecuencia de la constante actividad sísmica referida, el Estado de Chile y sus agencias técnicas, particularmente ONEMI y SHOA, se han enfocado en la elaboración de mapas de riesgo y evacuación actualizados para todas las ciudades y pueblos costeros del país. Todos estos mapas de evacuación presentan los mismos tres elementos fundamentales: rutas de eva- cuación, áreas de seguridad y la definición de una línea de seguridad ${ }^{7}$.

Las rutas de evacuación son las vías y calles que debiesen ser utilizadas para evacuar hacia áreas seguras en caso de desastres. Las áreas de seguridad son lugares localizados sobre la altura mínima de seguridad definida por las autoridades donde la comunidad debiera reunirse en caso de desastres para organizarse, esperar información y ayuda por parte de autoridades y otras organizaciones de socorro. Finalmente, la línea de seguridad es una línea ficticia, paralela a la costa, que sirve como límite entre la zona segura y la zona vulnerable de la ciudad en caso de tsunami.

La red de seguridad urbana existente en lquique (Figura 2), según ha sido definida por las autoridades, consiste de 43 vías de evacuación y 68 áreas de seguridad, según consta en el plano de seguridad elaborado por la Oficina Nacional de Emergencia (ONEMI) en 2017.

Este plano fue concebido por el SHOA en base a simulaciones numéricas de tsunamis, utilizando el sistema COMCOT, a partir de la información disponible del terremoto de 1877 (Castro et al., 2015). Esta red de seguridad debiera dar acogida a una población afectada de 108,881 personas (León y March, 2016). 


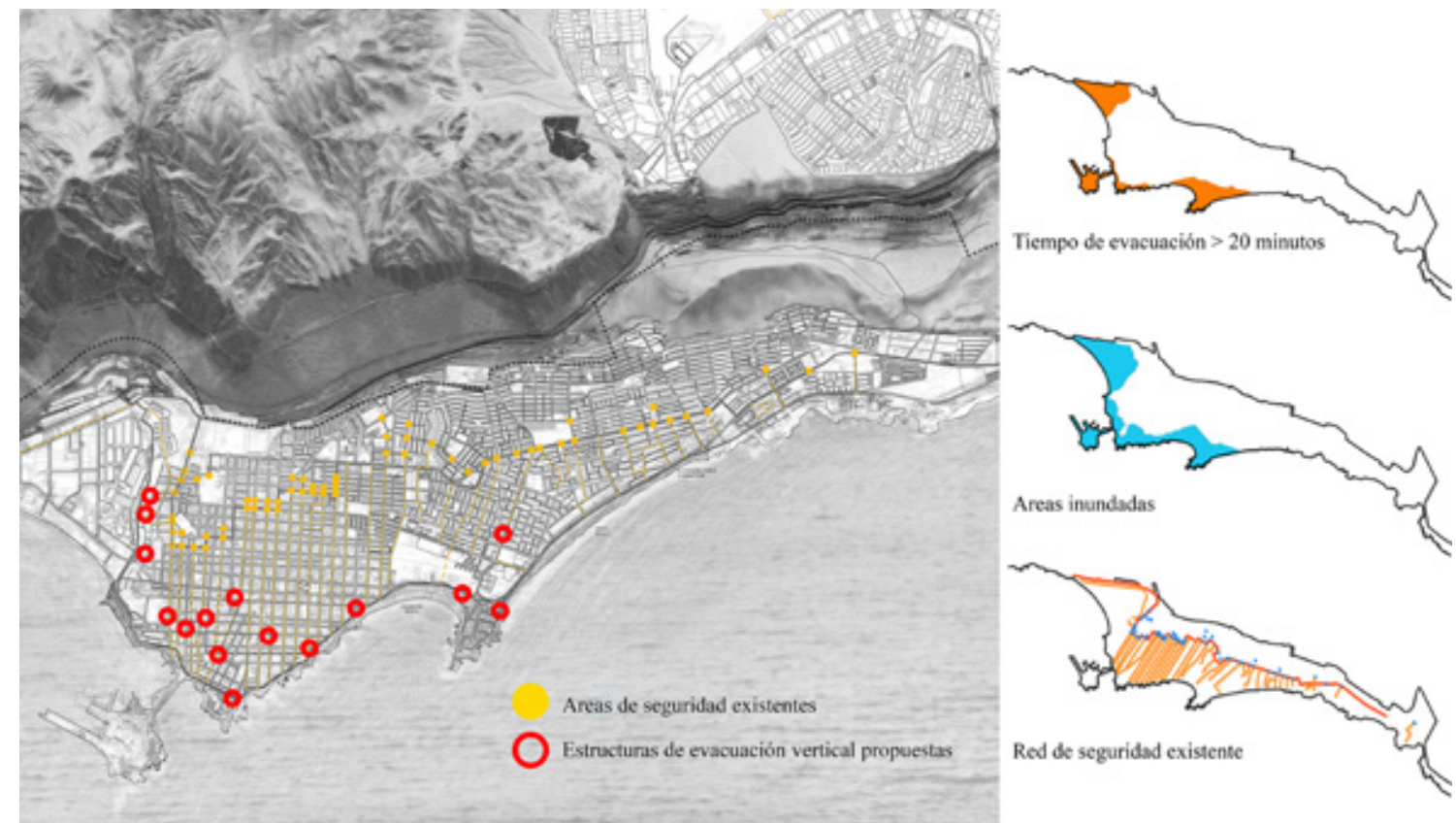

Figura 3 Red propuesta de estructuras para evacuación vertical. Fuente: Elaboración de los autores.

En su estado actual, la red de seguridad urbana presenta dos problemas. Primero, en algunos sectores la distancia entre la costa y las áreas de seguridad es extremadamente larga $y$, segundo, los sectores definidos como áreas de seguridad no cuentan con ningún tipo de equipamiento o refugio para recibir de manera apropiada a la población en estado de evacuación.

\section{Áreas potencialmente inundables}

Con respecto a la estimación de posibles daños generados por tsunamis en la ciudad de lquique, algunos estudios recientes han propuesto simulaciones que logran mostrar de manera clara y precisa las posibles consecuencias de un terremoto y tsunami de gran escala en la ciudad.

Para este artículo se emplea como ejemplo, el proyecto "SATREPS Chile Tsunami Project" desarrollado por la "Science and Technology Research Partnership for Sustainable Development" (SATREPS) y la "Japan International Cooperation Agency" (JICA) ${ }^{8}$, en el año 2016. En este proyecto, una serie de simulaciones para eventos de tsunami en Iquique fueron realizadas utilizando el software TUNAMI N2, desarrollado por la Universidad de Tohoku, Japón.

Dichas simulaciones consideraron 3 posibles escenarios: Escenario $\mathrm{A}(8.8 \mathrm{Mw})$, escenario $\mathrm{B}(9.0 \mathrm{Mw})$ y el escenario $C(8.9 \mathrm{Mw})$. Estos escenarios están, a su vez, basados en estudios previos realizados por Yagi et al. (2014), que propuso los escenarios de ruptura, y Chlieh et al. (2011), que calculó los potenciales sísmicos (SATREPS, 2016).

Cabe mencionar que los resultados de las simulaciones de estos tres escenarios revelan serios daños de inundación a lo largo del área costera de la ciudad (Figura 3). Los escenarios $\mathrm{A}(8.8 \mathrm{Mw})$ y $\mathrm{C}(8.9 \mathrm{Mw})$ presentan profundidades de inundación máximas de entre 2 y 5 metros en áreas específicas, además de inundaciones generalizadas de entre 0.5 y 2 metros. El peor posible escenario es $B(9.0 \mathrm{Mw})$, con inundaciones mucho más extendidas, de entre 2 y 5 metros de profundidad e inundaciones máximas de entre 5 y 10 metros de profundidad en áreas específicas. En ambos casos, las áreas más afectadas serían el sector de ZOFRI, la península, el puerto y, en menor medida, el casco histórico. 
De acuerdo a las simulaciones llevadas a cabo en este estudio, la primera ola de tsunami alcanzaría la costa después de tan solo 20 minutos, y una segunda y tercera ola, lo harían 60 minutos después del terremoto.

\section{Tiempos potenciales de evacuación}

A través de la simulación de estos diversos escenarios, ya es posible constatar cómo extensas áreas de la ciudad de lquique se verían seriamente afectadas en caso de tsunami. Con esta información, es también importante analizar la performance de tiempos de evacuación para un evento de tsunami de gran escala.

Con el fin de analizar precisamente la performance de evacuación ante tsunami de la ciudad de lquique, se toma como referencia una serie de simulaciones efectuadas para estimar los tiempos de evacuación en caso de un tsunami de gran escala por León y March (2016). Esta investigación considera el uso de dos modelos computacionales distintos, los cuales se detallan a continuación.

El primer modelo está enfocado en analizar la configuración urbana de la ciudad, es decir, de qué manera los distintos espacios de evacuación (calles, plazas, pasajes, etc.) están conectados unos con otros y cómo éstos pueden conducir de manera exitosa a la población desde sus lugares hasta terreno seguro.

El segundo modelo desarrollado es un modelo basado en agentes. El resultado de esta simulación, después de una serie de iteraciones en un lapso determinado, permite calcular el tiempo requerido para mover a todos los agentes desde un área de riesgo de tsunami a un área segura. En este modelo a cada agente evacuado se le otorga una ruta óptima para seguir, una velocidad de evacuación $(1.4 \mathrm{~m} / \mathrm{s})$ y tres factores de reducción de velocidad: pendiente del terreno, características de las calles y condiciones de tráfico y, por último, cantidad de evacuados: a mayor cantidad de evacuados, mayor es la dificultad de movimiento.

El estudio de estos dos modelos, realizados por León y March, comprueban que la ciudad de lquique posee serios problemas con respecto a sus tiempos de evacuación (Figura 3), en ocasiones incluso sobrepasando los 35-40 minutos, debido a las distancias entre la costa y las áreas de seguridad (León y March, 2016.

Al igual que en el estudio anterior, las áreas más críticas resultan ser la ZOFRI, la península, Cavancha y el puerto.

\section{RESULTADOS}

A través del estudio y análisis de los diversos escenarios simulados en estas investigaciones anteriores se puede observar cómo extensas áreas de gran importancia para la ciudad de lquique se verían profundamente afectadas en caso de tsunami.
Adicionalmente, se aprecia que la performance en términos de evacuación frente a este tipo de eventos presenta algunos problemas considerables, fundamentalmente relacionados con tiempos de evacuación excesivos y zonas de seguridad con equipamientos deficientes.

Tomando en consideración estos antecedentes, parece importante considerar las posibles maneras de mejorar la respuesta de la ciudad ante eventos de tsunami desde el punto de vista del diseño arquitectónico. Entendiendo las extensas zonas que se verían afectadas y los excesivos tiempos de evacuación, se vuelve pertinente contemplar la adición de un nuevo componente a la red urbana de seguridad con el propósito de mejorar su respuesta: Un sistema de estructuras de evacuación vertical (Figura 4).

\section{Mejoras a la red urbana de seguridad}

En caso de tsunamis, la primera prioridad es siempre la evacuación horizontal hacia terrenos altos; pero esto no es siempre posible debido a las largas distancias desde la costa hasta las áreas seguras y a los tiempos de advertencia que a veces son muy cortos, especialmente en los casos de tsunami con orígenes cercanos a la costa. En dichas ocasiones, refugiarseen-el-lugar o refugiarse-cerca-del-lugar a través del uso de estructuras de evacuación vertical puede ser una alternativa válida para escapar de los riesgos del fenómeno (Federal Emergency Management Agency, 2009).

Actualmente, en Chile no existen ejemplos de este tipo de estructuras. Por el contrario, hay numerosos ejemplos exitosos de su aplicación en países como Japón (Figura 5) y Estados Unidos (sobre todo en estados de la costa oeste como Washington y Oregón), además de naciones del sudeste asiático como Indonesia, en particular, la región de Banda Aceh, que fue arrasada después del terremoto y tsunami del Océano Índico, en el año 2004. (Yuzal et al., 2017).

Concretamente en Japón, existen desde 1982 precedentes de estructuras de evacuación vertical llamadas "tsunami-hinan", ubicadas en la localidad de Kesennuma. Durante el gran terremoto de 2011, en la región de Tohoku, las estructuras de evacuación vertical dieron refugio a más de 5,400 personas, distribuidas en 37 refugios (Fraser et al., 2012: 446).

De la experiencia internacional, en términos de estructuras de evacuación vertical, se desprenden ciertos requerimientos técnicos universales para éstas, como resistencia estructural, planificación de la localización, accesibilidad, señalética, altura y número de pisos (ibidem, 448).

Considerando las caracteristicas particulares de lquique y sus requerimientos, se proponen los siguientes tres lineamientos de diseño como complemento a las definiciones técnicas explicadas anteriormente. 
PREPRINT

AS54

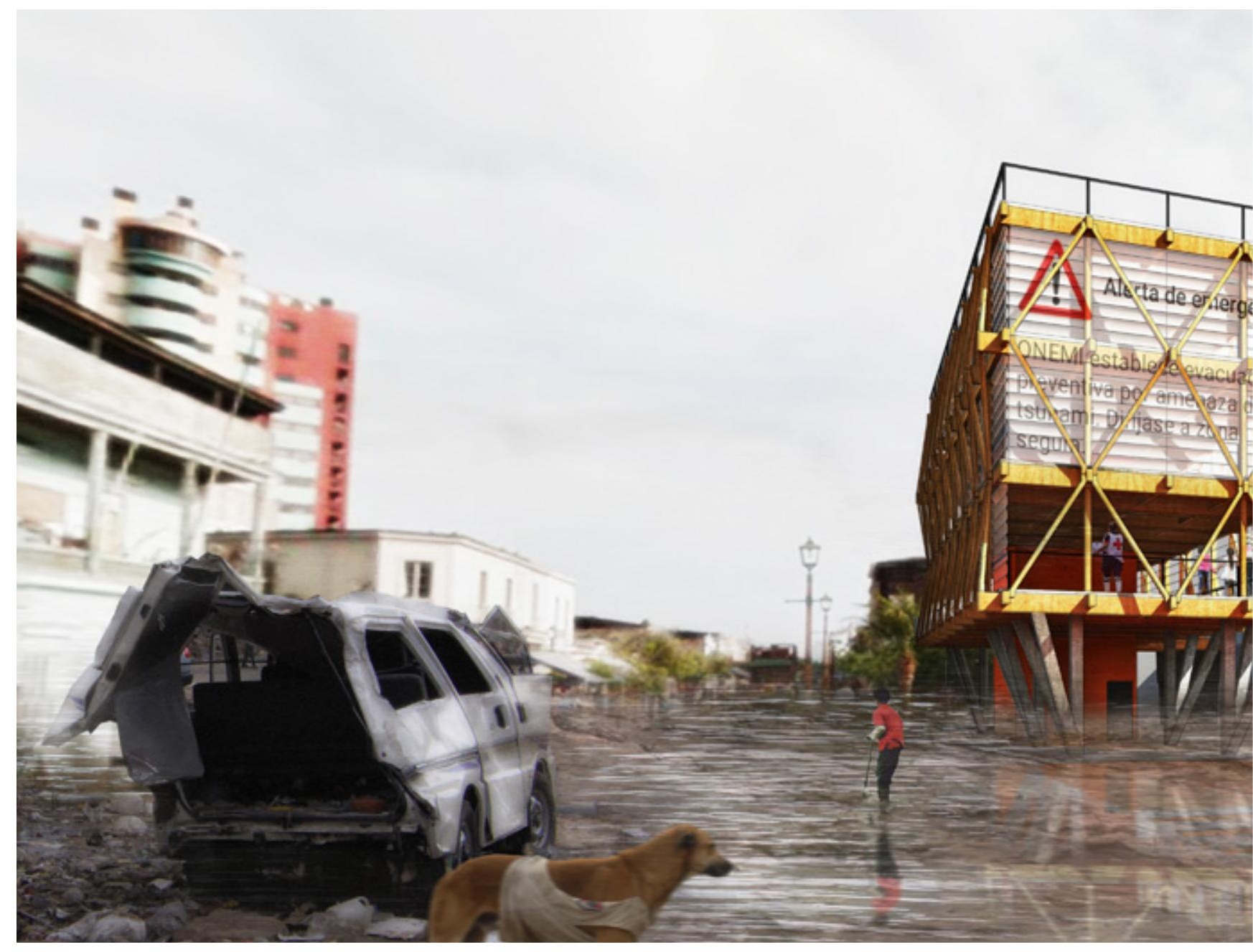

Figura 4 Vista de prototipo de evacuación vertical en situación de tsunami. Fuente: Elaboración de los autores. 
PREPRINT A554

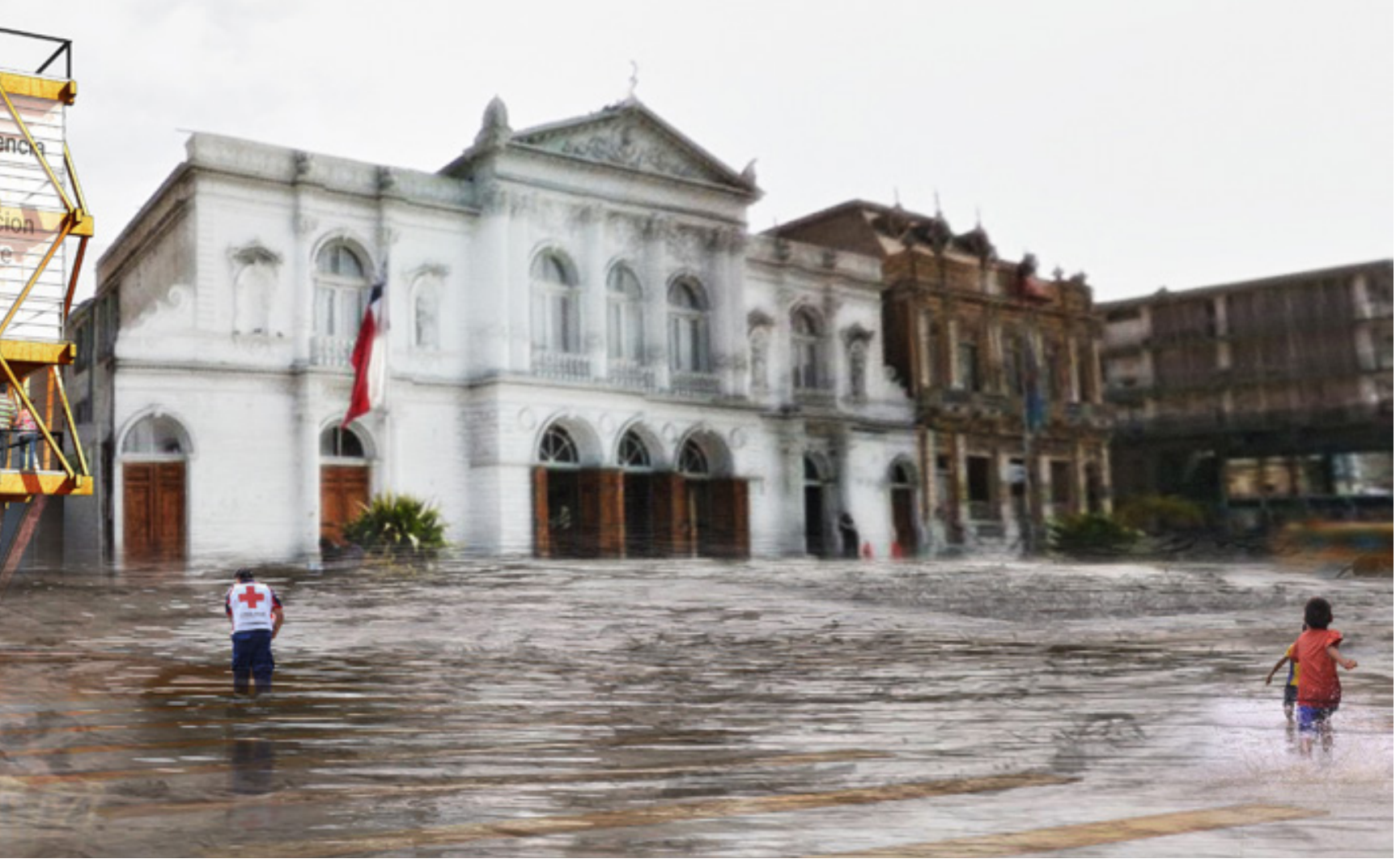




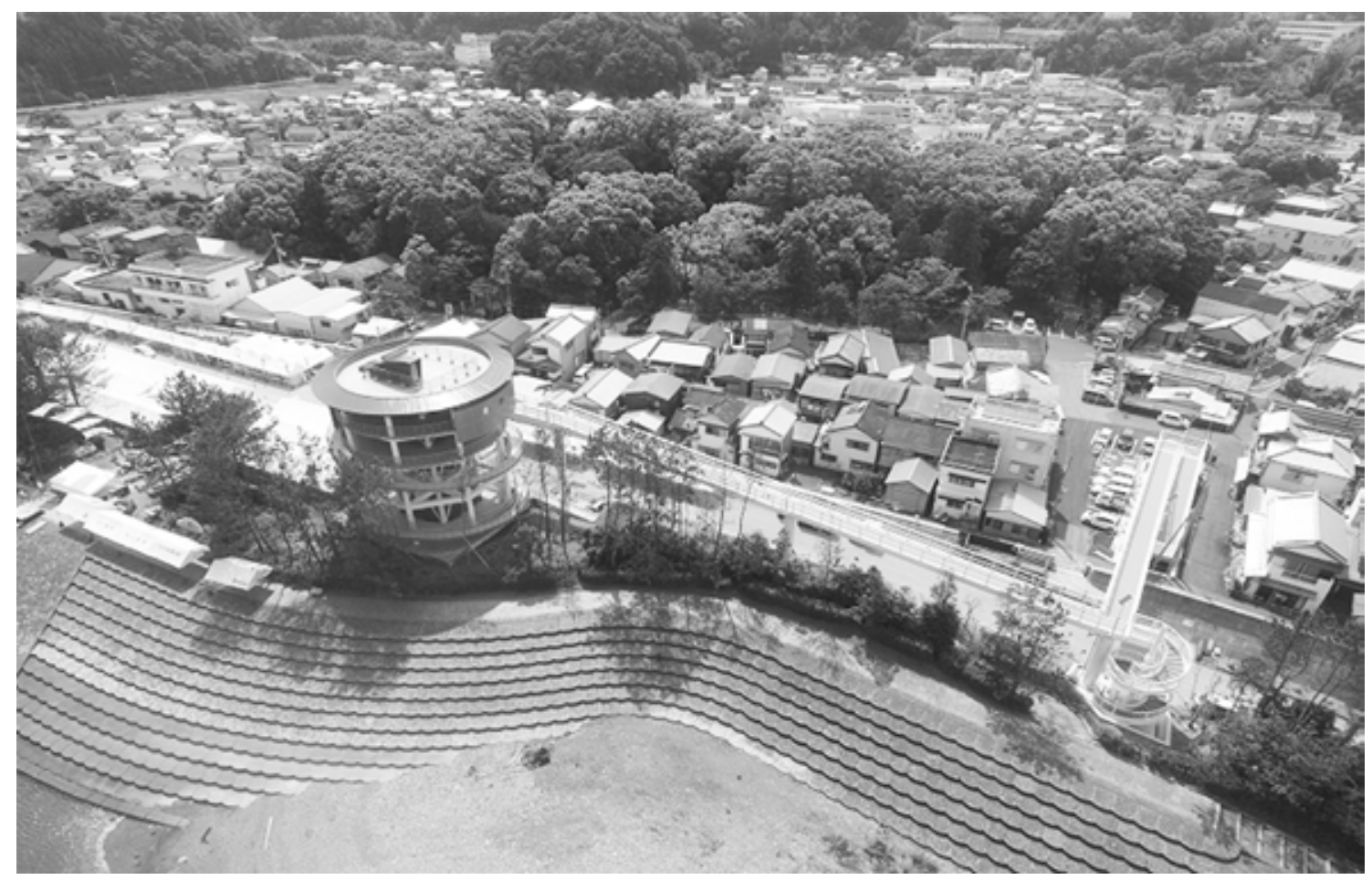

Figura 5 Vista de torre de evacuación de tsunami en Kochi, Japón. Fuente: Fotografía de Masahiro Kusumoto. (www.g-mark.org, viewed 09-08-2018 http://www.g-mark.org/award/describe/44371?locale=en).

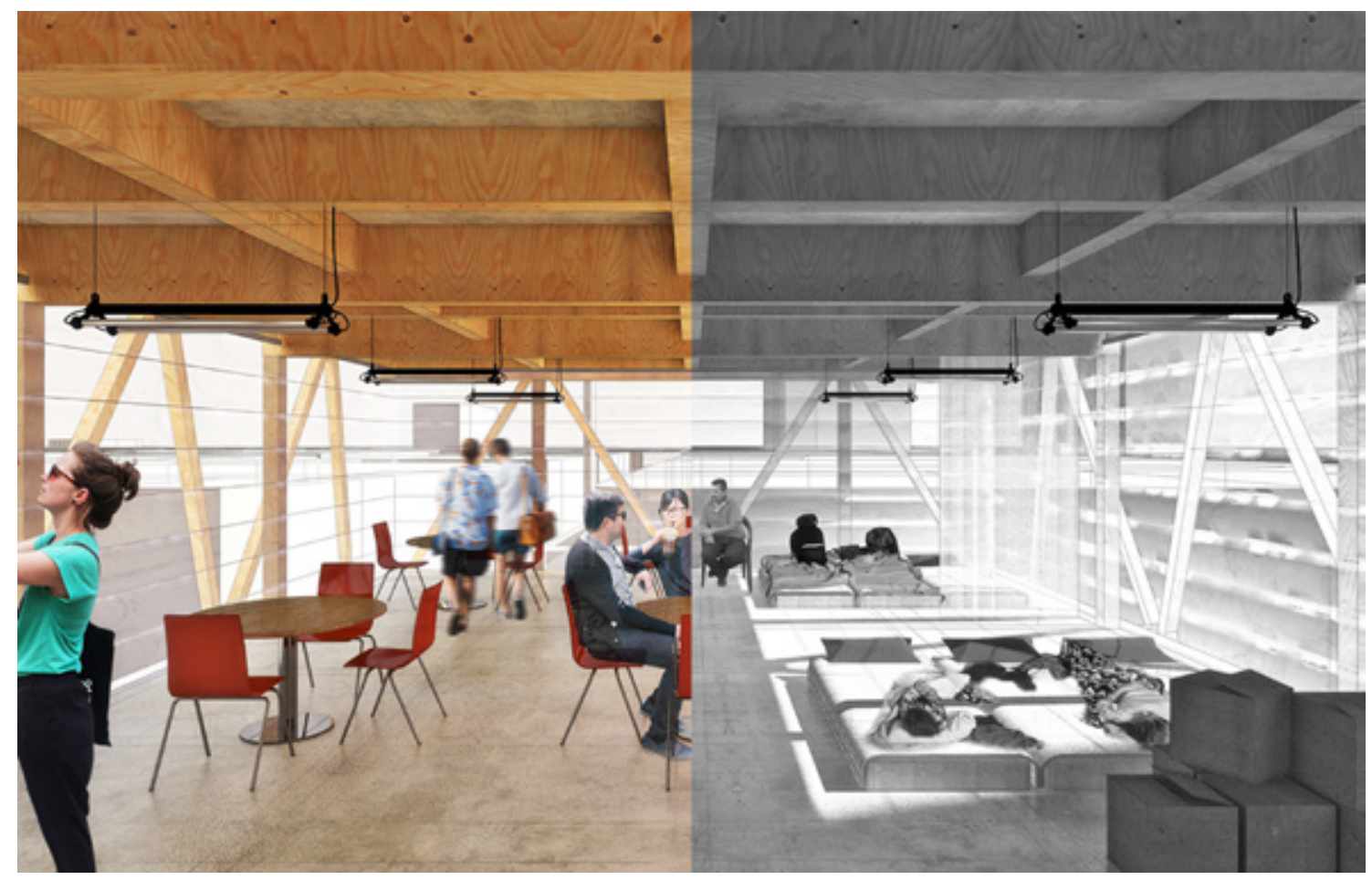

Figura 6 Vista de espacio interior que muestra dualidad de uso entre situaciones de vida diaria (izquierda) y de emergencia (derecha). Fuente: Elaboración de los autores. 
Flexibilidad y dualidad:

Una de las principales consideraciones cuando se diseñan estructuras de evacuación vertical es el problema del uso, por cuanto resulta imperante buscar la manera de promover una mayor intensidad de uso, a pesar de que el programa central sea solo requerido en ocasiones puntuales de desastres naturales. Resulta clave entonces entender la necesidad de espacios flexibles que puedan modificar su uso según sea requerido (Figura 6), albergando, así, una diversidad de usos, no solo orientados a situaciones de desastre, sino también a la vida diaria, de manera que estas estructuras sean parte integral del tejido urbano y de la vida de sus usuarios.

La variedad de usos de estas estructuras en instancias de vida diaria debiera estar fuertemente ligada a su contexto inmediato, concretándose en centros comunitarios, centros de información turística, actividades religiosas, culturales, entre otras, que permitan apropiación e identificación por parte de la comunidad circundante; una característica vital a la hora de planificar estructuras de evacuación vertical (ibidem, 454).

Independencia y sustentabilidad:

Otro problema importante de las áreas de seguridad existentes en la ciudad es la falta de refugio y servicios higiénicos, en tanto estas áreas son tan solo espacios abiertos sin ningún tipo de programa. Siguiendo los últimos lineamientos definidos por el gobierno, las estructuras de evacuación vertical deben proveer a los evacuados las condiciones mínimas de confort, higiene y refugio, al menos para las primeras 12 horas después de ocurrido el desastre (Ministerio de Vivienda y Urbanismo et al., 2017). Al mismo tiempo, debe contemplarse la necesidad de contar con autosustentabilidad en términos energéticos e higiénicos a través del uso de recursos renovables, independientes de las redes domiciliarias de agua y electricidad.

Intervenciones puntuales y de escala reducida:

Como se ha mencionado previamente, la ciudad de lquique no ha sido afectada por eventos de tsunami de gran magnitud en las últimas décadas, por tanto, es una ciudad plenamente funcional $y$, como tal, se hace difícil proponer intervenciones de gran escala, debido a la falta de espacio y la densidad del tejido urbano existente. Para enfrentar este problema, las estructuras de evacuación debieran mantener una escala reducida para lograr trabajar en los intersticios y vacíos de pequeña escala, a modo de una intervención de acupuntura urbana (Figura 7).

\section{CONCLUSIONES}

Recapitulando, este artículo ha intentado alcanzar tres objetivos principales. Primero, entender y establecer una visión clara con respecto a la realidad chilena en términos de terremotos y tsunamis, asumiendo su recurrencia en el tiempo y su particular influencia el de- sarrollo de la arquitectura nacional y sus regulaciones. Luego, se buscó comprender la situación actual de la ciudad de lquique como caso de estudio y su posición con respecto a potenciales desastres futuros, en forma de terremotos y tsunami de gran escala. En tal sentido, se analizó la ciudad utilizando como punto de partida una serie de investigaciones previas que han permitido simular y estimar la hipotética respuesta de la ciudad en caso de un tsunami de gran escala.

Finalmente, después de lograr establecer una apreciación general de la respuesta de lquique y su red de seguridad urbana existente en caso de tsunami, esta investigación intentó aproximarse a potenciales mejoras al sistema, desde una perspectiva enfocada en el diseño arquitectónico, específicamente a través de la incorporación y el diseño de una red de estructuras de evacuación vertical capaz de reducir los tiempos de evacuación para la población localizada en las zonas más vulnerables.

Al tratarse de una ciudad que no se ha visto afectada por desastres de tsunami en las últimas décadas, lquique presenta un desafío interesante con respecto a cómo intervenir y diseñar dentro de un contexto urbano que se encuentra activo y funcionando, sin el "beneficio" de la tabula rasa post-desastre, lo cual hace posible proyectar en cualquier lugar y en cualquier escala. Para enfrentar este desafío, la investigación propone 3 recomendaciones de diseño que podrían contribuir a generar una propuesta arquitectónica efectiva: usos duales y flexibilidad programática, autosustentabilidad, e intervenciones puntuales de escala reducida.

Trabajar de manera integrada entre el tejido urbano y establecer relaciones cercanas con la ciudad existente, parecen ser la clave para una intervención exitosa, que pueda mejorar la situación actual de Iquique, no solo para tiempos de desastres naturales y emergencia, sino también para acrecentar el estándar de vida general para la ciudad y sus habitantes. 


\section{PREPRINT}

AS54

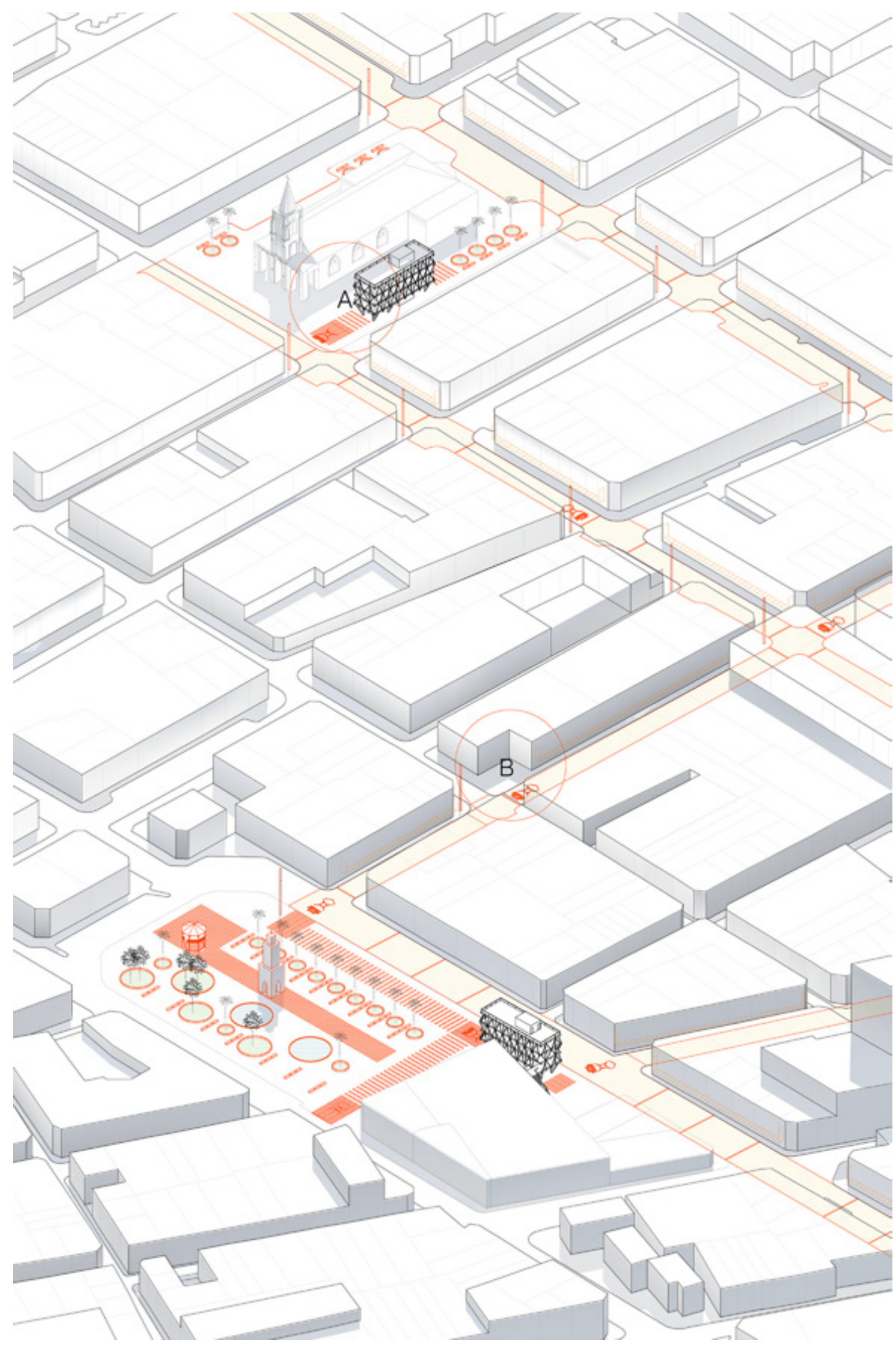

Figura 7 Axonométrica de los prototipos dispuestos intersticialmente entre el tejido urbano existente. Fuente: Elaboración de los autores. 


\section{REFERENCIAS BIBLIOGRÁFICAS}

BAERISWYL, Sergio. Resiliencia urbana; aprender a habitar con las amenazas de la naturaleza. La experiencia del terremoto y tsunami de 2010 en las costas del BíoBío, Revista Márgenes, 2014, vol. 11, n¹5, pp. 7-16.

BARRIENTOS, Sergio. Earthquakes in Chile. En: MORENO, Teresa y GIBBONS, Wes. The Geology of Chile. Londres: The Geological Society of London, 2007, pp. 263-289.

CASTRO, Carmen Paz; IBARRA, Ignacio; LUKAS, Michael; ORTIZ, Jorge y SARMIENTO, Juan Pablo. Disaster risk construction in the progressive consolidation of informal settlements: Iquique and Puerto Montt (Chile) case studies, International Journal of Disaster Risk Reduction, 2015, vol.13, pp. 109-127.

CHLIEH, Mohamed; PERFETTINI, Hugo; TAVERA, Hernán; AVOUAC, Jean Philippe; REMY, Dominique; NOCQUET, Jean Mathieu; ROLANDONE, Frederique; BONDOUX, Francis; GABALDA, Germinal y BONVALOT, Sylvain. Interseismic coupling potential along the Central Andes subduction zone, Journal of Geophysics Research [en línea], 2011, vol. 116, B12405, DOI:10.1029/2010JB008166.

FEDERAL EMERGENCY MANAGEMENT AGENCY. Vertical Evacuation from Tsunamis: A Guide for Community Officials. FEMA P646A, 2009.

FRASER, Stuart; LEONARD, Graham; MURAKAMI, Hitomi y MATSUO, Ichiro. Tsunami Vertical Evacuation Buildings - Lessons for International Preparedness Following the 2011 Great East Japan Tsunami, Journal of Disaster Research, 2012, vol. 7, pp. 446-457.

GUROVICH, Alberto. La ciudad de Iquique, Revista de arquitectura, 2005, vol. 1, n 1, pp. 8-11.

HERRMAN, Marie. The role of Urban Planning in Mitigating Tsunami in Chile after February 27th, 2010, Revista de Urbanismo, 2016, n³ 34, pp. 20-33.

LEÓN, Jorge y MARCH, Alan. An urban form response to disaster vulnerability: Improving tsunami evacuation in Iquique, Chile, Environment and Planning B: Planning and Design, 2016, vol. 43, n 5, pp. 826-847.

MINISTERIO DE VIVIENDA Y URBANISMO; MINISTERIO DEL INTERIOR Y SEGURIDAD PÚBLICA; MINISTERIO DE ENERGÍA; OFICINA NACIONAL DE EMERGENCIAS y CENTRO NACIONAL DE INVESTIGACIÓN PARA LA GESTIÓN INTEGRADA DE DESASTRES NATURALES. Guía de referencia para sistemas de evacuación comunales por tsunami, 2017. Santiago de Chile.

PIZZI, Marcela. El revival griego en la arquitectura de Iquique, Revista de arquitectura, 2005, vol. 10, n 11, pp. $12-15$

SATREPS CHILE TSUNAMI PROJECT. Guía para la Estimación de Peligro de Tsunami. Research Project on Enhancement of Technology to Develop Tsunami-Resilient Community, 2015, Santiago de Chile.
SCHURR, Bernd; ASCH, Gunter; HAINZL, Sebastian; BEDFORD, Jonathan; HOECHNER, Andreas; PALO, Mauro; WANG, Rongjiang; MORENO, Mauro; BARTSCH, Mitja; ZHANG, Yong; ONCKEN, Onno; TILMANN, Frederick; DAHM, Torsten; VÍCTOR, Pia; BARRIENTOS, Sergio y VILOTTE, Jean-Pierre. Gradual unlocking of plate boundary controlled initiation of the 2014 Iquique earthquake, Nature [en línea], 2014, vol. 512, pp. 299-302. DOI:10.1038/nature13681.

YAGI, Yuji; TAKAHASHI, Tomoyuki; OKUMURA, Yoshihiro y ARÁNGUIZ, Rafael (2014). Tsunami hazard estimation: Case of Iquique. En: Seminar on Disaster Mitigation for Earthquake and Tsunami Countries of Latin America, november 2014, Santiago, Chile.

YUZAL, Hendri; KIM, Karl; PANT, Pandrip y YAMASHITA Eric. Tsunami evacuation buildings and evacuation planning in Banda Aceh, Indonesia, Journal of Emergency Management [en línea], 2017, vol. 15, n 1, pp. 49-61. DOI:10.5055/jem.2017.0312 